PROCEEDINGS OF THE

AMERICAN MATHEMATICAL SOCIETY

Volume 130, Number 7, Pages 1887-1892

S 0002-9939(02)06510-3

Article electronically published on February 8, 2002

\title{
TWISTED HIGHER MOMENTS OF KLOOSTERMAN SUMS
}

\author{
CHUNLEI LIU
}

(Communicated by David E. Rohrlich)

AbStract. Let $\chi$ be a nontrivial Dirichlet character modulo an odd prime $p$. Write

$$
S(a)=\sum_{x=1}^{p-1} e\left(\frac{x+a x^{-1}}{p}\right)=2 \sqrt{p} \cos \theta(a) .
$$

We shall prove

$$
\sum_{a=1}^{p-1} \chi(a) S(a)^{2}=\chi(-1) g(\chi)^{2} J\left(\chi, \bar{\chi}^{2}\right)
$$

and, for complex $\chi$,

$$
\left|\sum_{a=1}^{p-1} \chi(a) \frac{\sin (k+1) \theta(a)}{\sin \theta(a)}\right| \leq c(k) \sqrt{p}, k>0,
$$

where $c(k)$ is a constant depending only on $k$.

\section{INTRODUCTION}

Let $p$ be an odd prime. Write

$$
S(a)=\sum_{x=1}^{p-1} e\left(\frac{x+a x^{-1}}{p}\right) .
$$

It is a Kloosterman sum. It is known that ([I]

$$
\begin{gathered}
\sum_{a=1}^{p-1} S(a)=1, \\
\sum_{a=1}^{p-1} S(a)^{2}=p^{2}-p-1, \\
\sum_{a=1}^{p-1} S(a)^{3}=\left(\frac{-3}{p}\right) p^{2}+2 p+1
\end{gathered}
$$

Received by the editors September 19, 2000.

2000 Mathematics Subject Classification. Primary 11L05.

Key words and phrases. Kloosterman sum, Dirichlet character.

This research is supported by MCSEC and NSFC. 
and

$$
\sum_{a=1}^{p-1} S(a)^{4}=2 p^{3}-3 p^{2}-p-1 .
$$

By A. Weil's result $([\underline{\underline{W}}])$, we may write

$$
\cos \theta(a)=\frac{1}{2 \sqrt{p}} S(a) .
$$

$\operatorname{Katz}([\mathrm{K}])$ proved the following equidistribution result:

$$
\left|\sum_{a=1}^{p-1} \frac{\sin (k+1) \theta(a)}{\sin \theta(a)}\right| \leq \frac{k+1}{2} \sqrt{p}, k \geq 1 .
$$

We may expect, for any Dirichlet character $\chi$ to the modulus $p$, that

$$
\left|\sum_{a=1}^{p-1} \chi(a) \frac{\sin (k+1) \theta(a)}{\sin \theta(a)}\right| \leq \frac{k+1}{2} \sqrt{p}, k \geq 1 .
$$

D. H. and E. Lehmer ( $[\mathrm{L})$ found empirically in 1952 and proved in 1959 that

$$
\sum_{a=1}^{p-1}\left(\frac{a}{p}\right) \frac{\sin 4 \theta(a)}{\sin \theta(a)}=2 \sqrt{p}\left(\frac{-1}{p}\right)\left(A^{2} / p-1\right)
$$

if $p=A^{2}+3 B^{2}, 3 \mid(A+1)$ and vanishes if $3 \mid(p+1)$.

We shall prove

Theorem 1. If $\chi$ is a nonquadratic Dirichlet character to the modulus $p$, then

$$
\left|\sum_{a=1}^{p-1} \chi(a) \frac{\sin (k+1) \theta(a)}{\sin \theta(a)}\right| \leq c(k) \sqrt{p}, k>0,
$$

where $c(k)$ is a constant depending only on $k$.

If $k=2$, we can prove more.

\section{Theorem 2.}

$$
\sum_{a=1}^{p-1} \chi(a) \frac{\sin 3 \theta(a)}{\sin \theta(a)}=\frac{1}{p} \chi(-1) g(\chi)^{2} J\left(\chi, \bar{\chi}^{2}\right),
$$

where $\chi$ is a nontrivial Dirichlet character to the modulus $p$,

$$
g(\chi)=\sum_{x=1}^{p-1} \chi(x) e\left(\frac{x}{p}\right)
$$

is a Gauss sum and

$$
J\left(\chi_{1}, \chi_{2}\right)=\sum_{x=1}^{p-1} \chi_{1}(x) \chi_{2}(1-x)
$$

is a Jacobi sum. 
Another form of Theorem 2 is

$$
\sum_{a=1}^{p-1} \chi(a) S(a)^{2}=\chi(-1) g(\chi)^{2} J\left(\chi, \bar{\chi}^{2}\right)
$$

where $\chi$ is a nontrivial Dirichlet character to the modulus $p$. It is equivalent to

$$
(p-1) S(a)^{2}=p^{2}-p-1+\sum_{\chi} \chi(-1) g(\chi)^{2} J\left(\chi, \bar{\chi}^{2}\right) \bar{\chi}(a),
$$

where $\chi$ runs over all nontrivial Dirichlet characters to the modulus $p$. So

$$
(p-1) S\left(a^{2}\right)^{2}=p^{2}-p-1+\sum_{\chi} \chi(-1) g(\chi)^{2} J\left(\chi, \bar{\chi}^{2}\right) \bar{\chi}^{2}(a),
$$

where $\chi$ runs over all nontrivial Dirichlet characters to the modulus $p$. It implies

$$
\left|\sum_{a=1}^{p-1} \chi(a) S\left(a^{2}\right)^{2}\right| \leq 2 p^{3 / 2}
$$

where $\chi$ is a nontrivial Dirichlet character to the modulus $p$. That is a little sharper than

$$
\left|\sum_{a=1}^{p-1} \chi(a) S\left(a^{2}\right)^{2}\right| \leq 4 p^{3 / 2}
$$

which was proved for quadratic Dirichlet character $\chi$ to the modulus $p$, and conjectured for general nontrivial Dirichlet character $\chi$ in $([\mathbf{C}])$ by Conrey and Iwaniec.

Remark. Theorems 1 and 2 generalize to the finite field case.

2. The TWisted SqUARE MOMENT

We now prove Theorem 2. Opening $S(a)^{2}$, we get

$$
\sum_{a=1}^{p-1} \chi(a) S(a)^{2}=\sum_{a=1}^{p-1} \chi(a) \sum_{x=1}^{p-1} \sum_{y=1}^{p-1} e\left(\frac{x+y}{p}\right) e\left(\frac{a\left(x^{-1}+y^{-1}\right)}{p}\right) .
$$

Summing over $a$ first and applying

$$
\sum_{a=1}^{p-1} \chi(a) e\left(\frac{a x}{p}\right)=\bar{\chi}(x) g(\chi),
$$

we get

$$
\sum_{a=1}^{p-1} \chi(a) S(a)^{2}=g(\chi) \sum_{x=1}^{p-1} \sum_{y=1}^{p-1} e\left(\frac{x+y}{p}\right) \bar{\chi}\left(x^{-1}+y^{-1}\right) .
$$

A change of variable yields

$$
\sum_{a=1}^{p-1} \chi(a) S(a)^{2}=g(\chi) \sum_{x=1}^{p-1} \sum_{y=1}^{p-1} e\left(\frac{x+x y}{p}\right) \bar{\chi}\left(x^{-1}+x^{-1} y^{-1}\right) .
$$

Summing over $x$ first and applying

$$
\sum_{a=1}^{p-1} \chi(a) e\left(\frac{a x}{p}\right)=\bar{\chi}(x) g(\chi)
$$


once more, we get

$$
\begin{array}{r}
\sum_{a=1}^{p-1} \chi(a) S(a)^{2}=g(\chi)^{2} \sum_{y=1}^{p-1} \bar{\chi}\left(1+y^{-1}\right) \bar{\chi}(1+y) \\
=g(\chi)^{2} \sum_{y=1}^{p-1} \chi(y) \bar{\chi}^{2}(1+y)=\chi(-1) g(\chi)^{2} J\left(\chi, \bar{\chi}^{2}\right) .
\end{array}
$$

\section{THE TWISTED HIGHER MOMENTS}

We now prove Theorem 1. For $q=p^{m}$, write

$$
S(a ; q)=\sum_{x} e\left(\frac{t r\left(x+a x^{-1}\right)}{p}\right)=2 \sqrt{q} \cos \theta(a ; q),
$$

where $x$ runs over all nonzero elements in $\mathbb{F}_{q}$, and $t r$ is the trace map from $\mathbb{F}_{q}$ to $\mathbb{F}_{p}$. Write

$$
T(\psi ; q)=\sum_{a} \psi(a) \frac{\sin (k+1) \theta(a ; q)}{\sin \theta(a ; q)},
$$

where $a$ runs over all nonzero elements in $\mathbb{F}_{q}$, and $\psi$ is a multiplicative character of $\mathbb{F}_{q}$. Write

$$
L(t)=\exp \left(\sum_{m \geq 1} T\left(\chi_{m} ; p^{m}\right) t^{m} / m\right)
$$

where $\chi_{m}$ is the lift of $\chi$ from $\mathbb{F}_{p}$ to $\mathbb{F}_{p^{m}}$. According to B. Dwork $\left.(\underline{\mathrm{Dw}}]\right), L(t)$ is a rational function

$$
L(t)=\prod_{v \in I}\left(1-\alpha_{v}(\chi) t\right) \prod_{v \in J}\left(1-\alpha_{v}(\chi) t\right)^{-1}
$$

Equivalently,

$$
T\left(\chi_{m} ; p^{m}\right)=-\sum_{v \in I} \alpha_{v}(\chi)^{m}+\sum_{v \in J} \alpha_{v}(\chi)^{m} .
$$

According to P. Deligne ([D] $)$,

$$
\left|\alpha_{v}(\chi)\right|=p^{l_{v} / 2}
$$

and the total number of $\alpha_{v}(\chi)$ is bounded by a number $c(k)$ depending only on $k$. We conclude that

$$
\left|\alpha_{v}(\chi)\right| \leq \sqrt{p}
$$

from which Theorem 2 follows. Otherwise, according to E. Bombieri's arguments $([\mathrm{B}]$,

$$
\left|T\left(\chi_{m} ; p^{m}\right)\right|>(1-\varepsilon) p^{m}
$$

for infinitely many $m$. Then

$$
\left|T\left(\chi_{m} ; p^{m}\right)\right|^{2}+\left|T\left(\bar{\chi}_{m} ; p^{m}\right)\right|^{2}>2(1-\varepsilon)^{2} p^{2 m}
$$

for infinitely many $m$, contradicting the following lemma. 


\section{Lemma 3.}

$$
\frac{1}{q-1} \sum_{\psi}|T(\psi ; q)|^{2}=q+O(\sqrt{q})
$$

where $\psi$ runs over all multiplicative characters of $\mathbb{F}_{q}$.

Indeed, we have

$$
\frac{1}{q-1} \sum_{\psi}|T(\psi ; q)|^{2}=\sum_{a}\left(\frac{\sin (k+1) \theta(a ; q)}{\sin \theta(a ; q)}\right)^{2} .
$$

As

$$
\left(\frac{\sin (k+1) \theta}{\sin \theta}\right)^{2}=1+\sum_{1 \leq l \leq 2 k} c_{l} \frac{\sin (l+1) \theta}{\sin \theta}
$$

we have

$$
\frac{1}{q-1} \sum_{\psi}|T(\psi ; q)|^{2}=q-1+\sum_{1 \leq l \leq 2 k} c_{l} \sum_{a} \frac{\sin (l+1) \theta(a ; q)}{\sin \theta(a ; q)}
$$

The lemma now follows from N. Katz's equidistribution result $([\underline{\mathrm{K}}])$

$$
\left|\sum_{a} \frac{\sin (l+1) \theta(a ; q)}{\sin \theta(a ; q)}\right| \leq \frac{l+1}{2} \sqrt{q}
$$

\section{ACKNOWLEDGMENT}

The author thanks J. Conrey and H. Iwaniec for offering their manuscript [C], N. Katz for sending his red book $[\mathrm{K}]$, Chengbiao Pan for his constant support, Kezheng Li for his willingness to help, Hongbin $\mathrm{Yu}$ and Fei $\mathrm{Xu}$ for informing the author about Lehmers' results, and Chungang Ji for exciting discussions.

\section{ADDED IN PROOF}

From Theorem 1 one can deduce that, for every odd integer $m$,

$$
\left|\sum_{a=1}^{p-1} \frac{\sin (k+1) \theta\left(a^{m}\right)}{\sin \theta\left(a^{m}\right)}\right| \leq(m, p-1) c(k) \sqrt{p}, k>0,
$$

where $c(k)$ is the constant in Theorem 1 . That is, for every fixed odd integer $m$, the angles $\theta\left(a^{m}\right)$, for $1 \leq a \leq p-1$, are equidistributed with respect to the Sato-Tate measure as $p$ goes to infinity.

\section{REFERENCES}

[B] E. Bombieri, On exponential sums in finite fields II, Invent. Math. 47 (1978), 29-39. MR 58:22072

[C] J. Conrey and H. Iwaniec, The cubic moment of central values of automorphic L-functions, Ann. Math. 151 (2000), 1175-1216. MR 2001g:11070

[D] P. Deligne, La conjecture de Weil I, Publ. Math. IHES 43 (1974), 273-307. MR 49:5013

[Dw] B. Dwork, On the rationality of the zeta function of an algebraic variety, Amer. J. Math. 82 (1960), 631-648. MR 25:3914

[I] H. Iwaniec, Topics in classical automorphic forms, GSM 17, AMS, Providence, Rhode Island, 1997. MR 98e:11051

[K] N. Katz, Gauss Sums, Kloosterman Sums and Monodromy Groups, Ann. Math. Studies 116, Princeton Univ. Press, 1988. MR 91a:11028 
[L] D. H. and E. Lehmer, On the cubes of Kloosterman sums, Acta Arith. 6 (1960), 15-22. MR 22:6773

[M] L. J. Mordell, On Lehmer's congruence associated with cubes of Kloostermann sums, J. London Math. Soc. 36 (1961), 335-339. MR 23:A3717

[W] A. Weil, On some exponential sums, Proc. Nat. Acad. Sci. USA 34 (1948), 204-207. MR 10:234e

Morningside Center of Mathematics, Chinese Academy of Science, Beijing 100080 , People's Republic of China

Current address: P. O. Box 1001-745, Zhengzhou 450002, People's Republic of China

E-mail address: chunleiliu@mail.china.com

$U R L$ : http://edtrchunleiliu.at.china.com 